\title{
Aortopexia toracoscópica para el tratamiento de la traqueomalacia grave en niños. Reporte de casos
}

\section{Thoracoscopic aortopexy to treat severe tracheomalacia in children. Cases report}

\section{Carlos García-Hernández ${ }^{1,2 *}$, Lourdes Carvajal-Figueroa ${ }^{1,2}$, Christian Archivaldo-García $^{1,2}$ y}

César M. Calderón-Alipi ${ }^{1,2}$

'Departamento de Cirugía Pediátrica, Hospital Infantil Privado; ${ }^{2}$ Curso Alta Especialidad, Cirugía Mínima Invasión Pediátrica, Universidad Autónoma de México. Ciudad de México, México

\section{Resumen}

Objetivo: Presentar nuestra experiencia en el tratamiento de la traqueomalacia grave por abordaje toracoscópico, la metodología diagnóstica, la técnica quirúrgica, la evolución postoperatoria y las complicaciones. Casos clínicos: De 2016 a 2017 se trataron tres pacientes, dos de ellos con traqueomalacia grave y uno con traqueobronquiomalacia grave, de 3 a 12 meses de edad, con peso de 3 a $6 \mathrm{~kg}$, múltiples eventos de casi muerte súbita y dependencia absoluta de ventilación asistida; en otro hospital practicaron traqueostomía, funduplicación y gastrostomía. La broncoscopia preoperatoria mostró malacia traqueal y bronquial grave, y la angiotomografía de tórax descartó otras causas de compresión. Se practicó aortopexia toracoscópica izquierda sin complicaciones, con control broncoscópico transoperatorio que mostró mejoría en la luz traqueal en los tres pacientes y sin recidiva de la sintomatología respiratoria en un seguimiento de 3 a 20 meses. Conclusiones: La aortopexia toracoscópica es una técnica segura y efectiva para el tratamiento de la traqueomalacia primaria grave en los niños.

PALABRAS CLAVE: Aortopexia. Toracoscopia. Traqueomalacia.

\begin{abstract}
Objective: To present our experience in the treatment of the severe tracheomalacia by thoracoscopic approach, as well as its diagnostic methodology, surgical technique, post-operatory evolution and complications. Clinical cases: From 2016 to 2017, three patients, 3, 9 and 12 months old, were treated. They showed multiple near sudden death events and had an absolute dependence to assisted ventilation. Pre-operatory bronchoscopy evinced tracheal severe malacia. Thoracic angiotomography discarded other extrinsic tracheal compression causes. A left thoracoscopic aortopexy was practiced with a bronchoscopical trans-operatory control; with improvement in tracheal space. Just one patient is still awaiting cannula removal, without symptomatology recurrence in a 3-20 months follow up. Conclusions: The thoracoscopic aortopexy is a safe and effective technique to treat primary and severe tracheomalacia in children.
\end{abstract}

KEY WORDS: Aortopexy. Thoracoscopy. Tracheomalacia.

\author{
Correspondencia: \\ *Carlos García-Hernández \\ Circuito Valle Dorado, 12 \\ Col. Loma de Valle Escondido \\ C.P. 52930, Atizapán de Zaragoza, Edo. de México, México \\ E-mail: carloscirped@ hotmail.com
}

Fecha de recepción: 01-10-2018

Fecha de aceptación: 16-04-2019

DOI: $10.24875 / C I R U .19000783$
Cir Cir. 2019;87:636-639 www.cirugiaycirujanos.com 


\section{Introducción}

La traqueomalacia es la debilidad de la tráquea, lo que favorece que se colapse con los cambios en la presión de la vía aérea ${ }^{1,2}$. Puede verse afectada toda la tráquea, solo un segmento e incluso los bronquios ${ }^{2-4}$.

La flacidez anormal de la tráquea incrementa la disminución fisiológica de la vía aérea que ocurre durante la espiración, y esta disminución de calibre es aún mayor cuando hay una espiración forzada, lo cual puede llevar a la obstrucción completa de la vía aérea ${ }^{5,6}$.

La traqueomalacia se clasifica en primaria o congénita, que se debe a una formación anormal del cartílago. La forma adquirida o secundaria es la más frecuente y está relacionada con otras lesiones torácicas ${ }^{2,7,8}$. La falta de una consistencia normal de la tráquea predispone a su colapso por compresión de las estructuras vasculares adyacentes en la cara anterior y en la cara posterior por el esófago, por lo que la deglución y el reflujo gastroesofágico agravan esta compresión $n^{9,10}$.

La sintomatología depende del grado de afectación de la tráquea y puede estar presente desde el nacimiento; Benjamin, et al. ${ }^{4}$ la clasifican en leve, moderada y grave ${ }^{4}$. Los casos graves presentan cianosis, estridor espiratorio o episodios de apnea grave que requieren ventilación asistida. La traqueomalacia grave está asociada con episodios de casi muerte súbita ${ }^{6,11}$. Ante esta sintomatología deben descartarse alteraciones esofágicas, cardiacas y neurológicas que pueden ocasionar casi muerte súbita ${ }^{12,13}$.

El tratamiento quirúrgico solo está indicado para los casos graves. La aortopexia es la piedra angular en el tratamiento de la traqueomalacia en pediatría. Esta técnica fue descrita por Filler y Fraga ${ }^{9}$ en 1976. Puede llevarse a cabo por una toracotomía izquierda o derecha, estereotomía parcial o cirugía de mínima invasión $n^{3,5,6,9}$. El primer abordaje por toracoscopia fue realizado por Decou et al. ${ }^{13}$ en 2001. Existen escasas publicaciones de este abordaje, y la serie más grande es la publicada por Van der Zee y Straver ${ }^{14}$ en 2015.

El objetivo de este artículo es comunicar la experiencia en el tratamiento de la traqueomalacia grave por abordaje toracoscópico, la técnica quirúrgica, la evolución posoperatoria y las complicaciones.

\section{Casos clínicos}

De enero de 2016 a julio de 2017 fueron tratados tres pacientes.

\section{Caso 1}

Masculino de 9 meses de edad, con un peso de $4 \mathrm{~kg}$, producto de gesta I, embarazo de 34 semanas, que inició su padecimiento desde el nacimiento con presencia de insuficiencia respiratoria, que requirió manejo con ventilación asistida, con imposibilidad para la extubación, motivo por el que se realizó una traqueostomía, con dependencia absoluta del ventilador. Al mes de vida se practicó gastrostomía con funduplicación de Nissen, con técnica abierta, motivo por el que se trasladó a nuestro hospital. Se realizó broncoscopia que mostro malacia traqueal grave. La angiotomografía de tórax fue normal. Con estos hallazgos se llevó a cabo una aortopexia anterior por abordaje toracoscópico izquierdo, con tres puertos de 5 y $3 \mathrm{~mm}$ (Fig. 1), presión de $5 \mathrm{mmHg}$, insuflando $0.1 \mathrm{l} / \mathrm{min}$. Se realizó disección mediastinal con retracción del lóbulo izquierdo del timo y se identificó el cayado aórtico hasta la emergencia del tronco braquiocefálico (Fig. 2). Se colocaron tres suturas de poliéster 2-0, de la aorta al esternón, la primera en el nacimiento del tronco braquiocefálico, la segunda en el cayado aórtico y la tercera en la emergencia de la aorta (Fig. 3); se anudaron con control endoscópico transoperatorio y se corroboró la apertura adecuada de la tráquea, con una mejoría del $80 \%$ en la luz traqueal. El tiempo quirúrgico fue de 120 minutos, sin sangrado y sin complicaciones posoperatorias. Se retiró la asistencia ventilatoria al décimo día del posoperatorio y se decanuló al séptimo mes del posoperatorio. Con un seguimiento de 20 meses, no hay datos de insuficiencia respiratoria.

\section{Caso 2}

Masculino de 12 meses de edad, con un peso de $6 \mathrm{~kg}$, producto de gesta II, embarazo de 38 semanas, que inició su padecimiento desde el nacimiento con presencia de insuficiencia respiratoria intermitente acompañada de estridor y múltiples episodios de casi muerte súbita, por lo que se practicaron funduplicación de Nissen y gastrostomía. Por la presencia de estridor constante, se practicó traqueostomía y requirió asistencia ventilatoria continua, por lo que se trasladó a nuestro hospital. Se realizó broncoscopia que mostró traqueobroncomalacia grave (Fig. 4). La angiotomografía de tórax fue normal. Con estos hallazgos, se realizó aortopexia con la técnica descrita, con una mejoría del $70 \%$ en la luz traqueal. El tiempo quirúrgico fue de 100 minutos, sin sangrado y sin complicaciones posoperatorias. Se retiró la asistencia ventilatoria al octavo día 


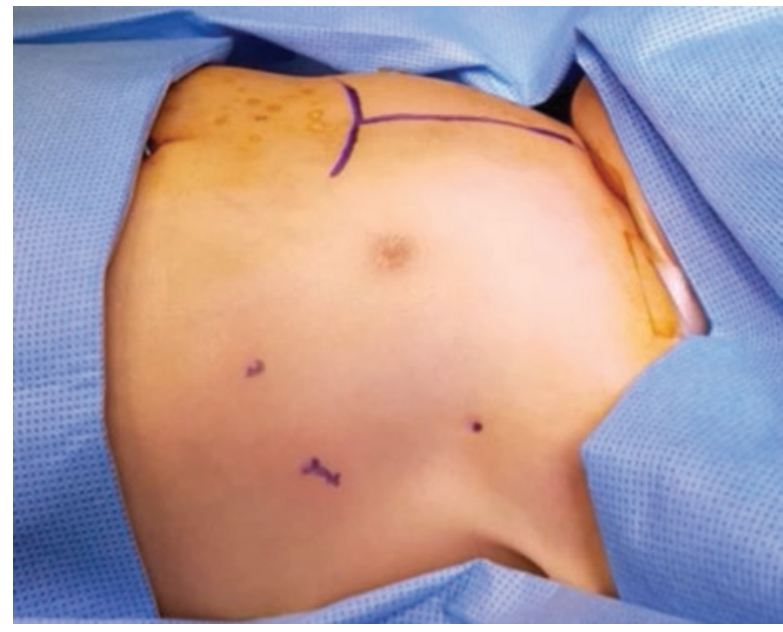

Figura 1. Colocación de los trocares y su relación con el esternón.

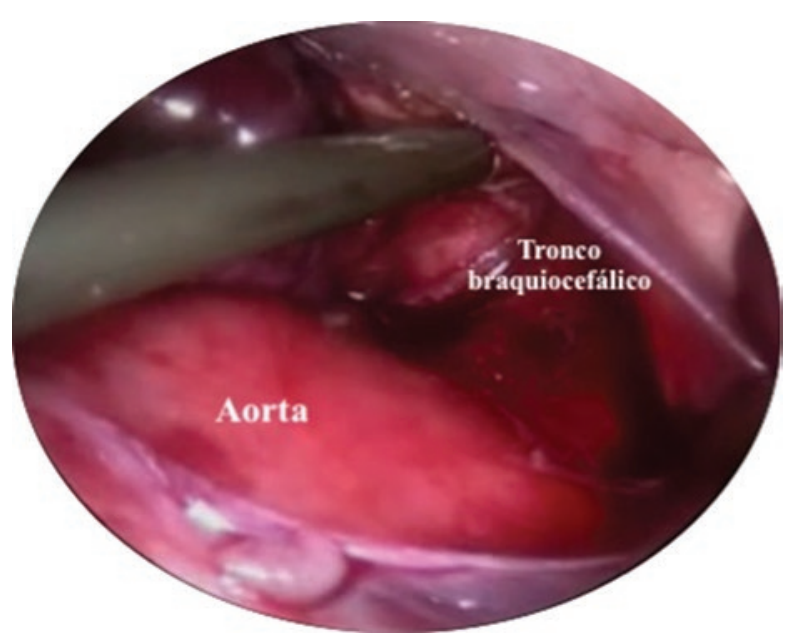

Figura 2. Visualización del arco aórtico, desde su nacimiento hasta la emergencia del tronco braquiocefálico. El lóbulo izquierdo del timo se encuentra retraído hacia la línea media.

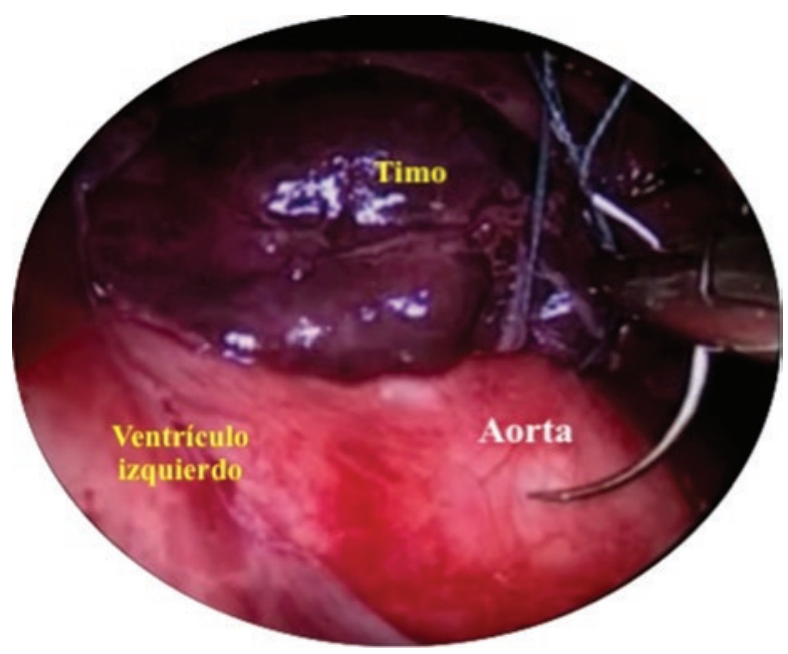

Figura 3. Tercera sutura, colocada lo más cercana posible a la emergencia de la aorta del ventrículo izquierdo.

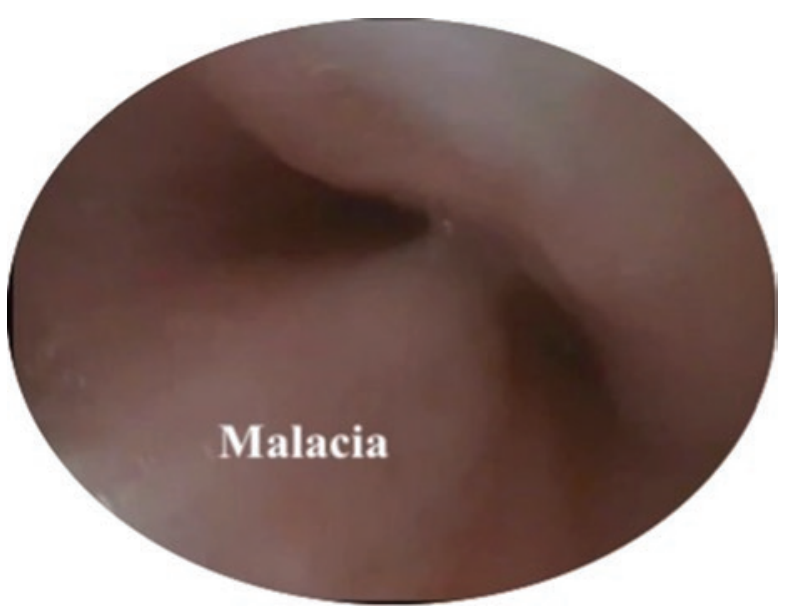

Figura 4. Aspecto broncoscópico de la gravedad de la malacia; las paredes anterior y posterior de la tráquea se encuentran adosadas.

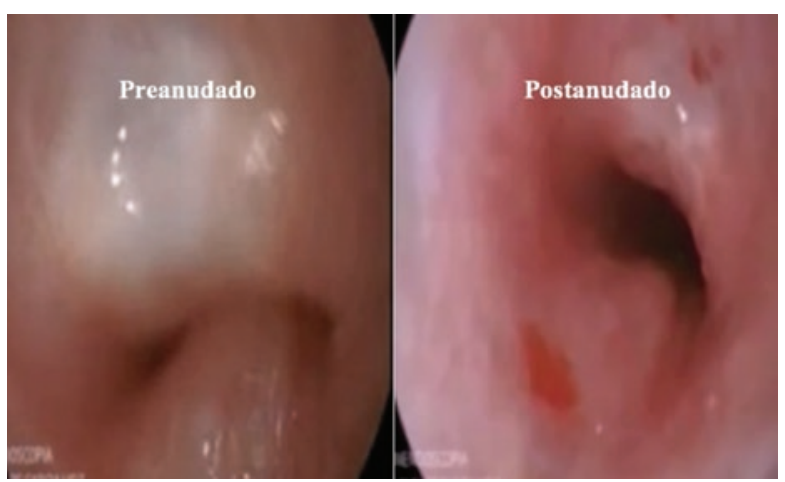

Figura 5. Aspecto endoscópico transoperatorio. A la derecha, antes de anudar las suturas, se evidencia la malacia grave con obstrucción total de la luz traqueal. A la izquierda, después de anudar las suturas, se aprecia una luz traqueal mayor del $80 \%$.

del posoperatorio y se decanuló al quinto mes del posoperatorio. Con un seguimiento de 12 meses, no hay datos de insuficiencia respiratoria.

\section{Caso 3}

Femenino de 3 meses de edad, con un peso de $3 \mathrm{~kg}$, producto de gesta I, embarazo de 36 semanas, que inició su padecimiento desde el nacimiento con presencia de estridor y múltiples episodios de casi muerte súbita, por lo que se practicaron funduplicación de Nissen y gastrostomía, así como traqueostomía a los 10 días de vida, y requirió asistencia ventilatoria continua, por lo que se trasladó a nuestro hospital. Se realizó broncoscopia que mostró traqueo malacia grave. La angiotomografía de tórax fue normal. Con estos hallazgos, se realizó aortopexia con la técnica descrita, con una mejoría del $80 \%$ en la luz traqueal (Figs. $5 \mathrm{~A}$ y B). El tiempo quirúrgico fue de 90 minutos, 
sin sangrado y sin complicaciones posoperatorias. Se retiró la asistencia ventilatoria al quinto día del posoperatorio. Aún no se ha decanulado y tiene un seguimiento de 3 meses, sin complicaciones.

\section{Discusión}

El tratamiento de la traqueomalacia depende de la gravedad de los síntomas y de los hallazgos endoscópicos $^{3,7}$. Solo los casos graves y cuando se haya descartado alguna otra patología que cause compresión en la tráquea requieren tratamiento quirúrgico específico, el cual puede ser resección de algún segmento traqueal, fijación de la pared posterior de la tráquea a la columna o colocación de cartílago autólogo².

La aortopexia es la mejor opción quirúrgica para la corrección de la traqueomalacia grave, pero habitualmente se requiere una esternotomía parcial o una toracotomía, lo que ha limitado su uso y ha hecho que se intente manejar a los pacientes con traqueostomías, gastrostomías y funduplicaciones ${ }^{2,3,5,6}$. Sin embargo, estos procedimientos no siempre consiguen el control de la sintomatología y de los eventos graves que ponen en peligro la vida, y requieren ventilación asistida, que es lo que ocurrió con nuestros pacientes. Por ello, fue necesario llevar a cabo una aortopexia y se consideró el abordaje toracoscópico como la mejor opción para nuestros pacientes. Para llevar a cabo este procedimiento es necesario hacer una exposición adecuada de la aorta desde su emergencia en el ventrículo izquierdo hasta visualizar la emergencia del tronco braquiocefálico, para poder colocar de manera cuidadosa las suturas necesarias para fijar la aorta al esternón y de esa manera evitar el colapso traqueal. El control endoscópico asegura el éxito del procedimiento, tal como lo pudimos ver en nuestros pacientes al mantener una luz traqueal adecuada para la ventilación espontánea, lo que permitió una rápida recuperación y poder retirar la ventilación asistida.

El abordaje toracoscópico requiere destrezas avanzadas en sutura y disección para disminuir el riesgo de las complicaciones.

Consideramos que esta técnica combina la posibilidad de resolver la traqueomalacia grave con las ventajas ya conocidas de la mínima invasión.

\section{Financiamiento}

Ninguno.

\section{Conflicto de intereses}

Los autores declaran que no existe conflicto de intereses.

\section{Responsabilidades éticas}

Protección de personas y animales. Los autores declaran que para esta investigación no se han realizado experimentos en seres humanos ni en animales.

Confidencialidad de los datos. Los autores declaran que han seguido los protocolos de su centro de trabajo sobre la publicación de datos de pacientes.

Derecho a la privacidad y consentimiento informado. Los autores han obtenido el consentimiento informado de los pacientes y/o sujetos referidos en el artículo. Este documento obra en poder del autor de correspondencia.

\section{Bibliografía}

1. Carden KA, Bosiselle PM, Waltz DA, Ernst A, Tracheomalacia and tracheobronchomalacia in children and adults. Chest. 2005:127:984-1005.

2. Fraga JC, Jennings RW, Kim PC. Pediatric tracheomalacia. Semin Pediatr Surg 2016;25:156-64

3. Blair GK, Cohen R, Filler RM. Treatment of tracheomalacia: eight years' experience. J Pediatr Surg. 1986;21:781-5.

4. Benjamin B, Cohen D, Glasson M. Tracheomalacia in association with congenital tracheoesophageal fistula. Surgery. 1976;79:504-8.

5. Kieley EM, Spitz L, Brereton R. Management of tracheomalacia by aortopexy. Pediatr Surg Int. 1987;2:13-5

6. Filler RM, Rossello P, Lebowitz RL. Life-threating anoxic spells caused by tracheal compression after repair of esophageal atresia: correction by surgery. J Pediatr Surg. 1976;11:739-48.

7. Boogard R, Huijsmans SH, Pijnenburg MWH, Tiddens HAWM, de Jongste JC, Merkus PJFM. Tracheomalacia and bronchomalacia in children: incidence and patient characteristics. Chest. 2005:128:3391-7.

8. Benjamin B. Tracheomalacia in infants and children. Ann Otol Rhinol Laryngol. 1984;93:438-42.

9. Filler RM, Fraga JC. Tracheomalacia. Semin Thorac Cardiovasc Surg. 1994;6:211-5.

10. Filler RM. Tracheomalacia. En: Fallis JC, Filler RM, Lemoine G, editores. Pediatric Thoracic surgery. New York, NY: Elsevier Science; 1991. p. 163-71.

11. Filler RM, Messineo A, Vinograd I. Severe tracheomalacia associated with esophageal atresia: results of surgical treatment. J Pediatr Surg. 1992;27:1136-41.

12. Filler RM. Tracheal congenital anomalies. En: Pearson EG, Deslauriers J, Hibert CA, Ginsber RJ, McKneally MF, Urschel HC, editores. Thoracic surgery. New York, NY: Churchill Livingstone; 1995. p. 235-50.

13. Decou JM, Parsons DS, Gauderer MWL. Thoracoscopic aortopexy for severe tracheomalacia. Pediatr Endosurg Innov Tech. 2001;5:205-8.

14. van der Zee DC, Straver M. Thoracoscopic aortopexy for tracheomalacia. World J Surg. 2015;39:158-64. 